\title{
ZBP1 is a significant pyroptosis regulator for systemic lupus erythematosus
}

\author{
Yan Huang ${ }^{1 \#}$, Dan-Dan Yang ${ }^{1 \#}$, Xiao-Ying Li $^{1}$, Da-Lang Fang ${ }^{2}$, Wei-Jie Zhou ${ }^{3,4}$ \\ ${ }^{1}$ Department of Rheumatology and Hematology, the People's Hospital of Baise, Baise, China; ${ }^{2}$ Department of Breast and Thyroid Surgery, The \\ Affiliated Hospital of Youjiang Medical University for Nationalities, Baise, China; ${ }^{3}$ Department of Clinical Laboratory, the People's Hospital of \\ Baisel, Baise, China; ${ }^{4}$ Center Laboratory of the People's Hospital of Baise, Baise, China \\ Contributions: (I) Conception and design: DD Yang; (II) Administrative support: DL Fang; (III) Provision of study materials or patients: DD Yang; (IV) \\ Collection and assembly of data: DL Fang; (V) Data analysis and interpretation: DD Yang; (VI) Manuscript writing: All authors; (VII) Final approval \\ of manuscript: All authors. \\ "These authors contributed equally to this work. \\ Correspondence to: Da-Lang Fang. Department of Breast and Thyroid Surgery, The Affiliated Hospital of Youjiang Medical University for \\ Nationalities, Baise, China. Email: fangdalang@stu.gxmu.edu.cn; Wei-Jie Zhou. Department of Clinical Laboratory, the People's Hospital of Baisel, \\ Baise, China. Email: zhouweijie1998@ymcn.edu.cn.
}

Background: Systemic lupus erythematosus (SLE) is a common autoimmune disease that affects all organs. Recently, several studies have shown that pyroptosis playsa significant process in the occurrence and progression of SLE. However, no study has investigated the association between pyroptosis genes and SLE. We conducted this study to examine this association.

Methods: The GSE11090, GSE20864, and GSE112087 gene microarrays of normal and SLE patient samples were downloaded from the Gene Expression Omnibus database. A differentially expressed gene (DEG) analysis was performed using the LIMMA package in R software. $\log _{2}$ fold change $\left|\log _{\mathrm{FC}}\right|>0.5$ and a false discovery rate (FDR) $<0.05$ setting for DEGs' screening value. We also performed an enrichment function analysis of the DEGs. To explore the role of pyroptosis genes in SLE, we selected pyroptosis genes that intersected with the DEGs for further analysis, we also examined the expression levels of the selected genes, their association with immune cell infiltration, and conducted western blotting and polymerase chain reaction analyses to confirm the selected genes expression levels in the SLE and normal samples.

Results: A total of 3,398 identical genes were obtained from 3 datasets for the differential analysis. 84 upregulated genes and 52 downregulated genes were identified in SLE. The enrichment function analysis revealed that DEGs act as key regulators of nicotinamide adenine dinucleotide (NADH) dehydrogenase activity, phospholipid scramblase activity, double-stranded ribonucleic acid (RNA) binding, and the interferon signaling pathway. We identified the SLE-related pyroptosis gene, Z-DNA binding protein 1 (ZBP1), by intersecting the DEGs of SLE and 40 pyroptosis genes. The differential analysis indicated that $Z B P 1$ was more highly expressed in SLE patients compared to normal samples $(\mathrm{P}<0.001)$. Additionally, the expression of $Z B P 1$ was higher in females than males $(\mathrm{P}=0.008)$. The SLE samples had different immune cell infiltration than the normal samples, and $Z B P 1$ was significantly correlated with immune cell infiltration in the SLE samples. Finally, the validation experiments results showed that ZBP1 expression levels were significantly more highly expressed in female and SLE patients, than male and normal patients.

Conclusions: ZBP1 may indicate that females have a high incidence rate of SLE, and it plays a significant role in the occurrence and progression of SLE.

Keywords: Pyroptosis; ZBP1; systemic lupus erythematosus (SLE)

Submitted Oct 25, 2021. Accepted for publication Dec 17, 2021.

doi: 10.21037/atm-21-6193

View this article at: https://dx.doi.org/10.21037/atm-21-6193 


\section{Introduction}

Systemic lupus erythematosus (SLE) is characterized by the loss of autoantibodies, massive immune complex deposition, the activation of inflammation, and immunity, which results in different degrees of damage in different organs (1). The main hallmarks of SLE are losing control of immune tolerance and the sustained production of autoantibodies against nuclear autoantigens (2). Research on the pathogenesis of SLE has shown that several types of programmed cell death play significant roles in the process of SLE (3). In 1972, Kerr et al. first defined programmed cell death apoptosis (4). Subsequently, several types of programmed cell death, such as autophagy, NETosis, necroptosis, and pyroptosis, have been defined and widely investigated in different diseases.

The dysregulation of cell death and a decrease in the ability to clear death cells lead to damage-associated molecular patterns, and enhance inflammation, immunity activation and the generation of autoantigens, resulting in tissue and organ damage in SLE patients (5). As programmed cell death plays a critical role in SLE, we focused on the role of pyroptosis-related genes in SLE. Pyroptosis is a type of programmed cell death that is characterized by cell lysis and inflammation induced by various damage signals (6). Its main features are gasdermin family-mediated cell deformation and eventual lysis, which results in the release of cell contents, including interleukin (IL-) $1 \beta$ and IL-18 (3). Pyroptosis was first observed in Shigella flexneri-infected macrophages (7). The activation of pyroptosis triggers the assembly of the inflammasome sensor, which leads to inflammation (8). Pyroptosis plays a key role in various diseases (9-12). In relation to SLE, research has shown that abnormal cell death and the dysregulation of dead cell clearance induces the production of antinuclear antibodies and other aberrant immune responses (13).

Pyroptosis is a type of cell death, and the dysregulation of pyroptosis has been implicated in the pathogenesis of lupus nephritis $(\mathrm{LN})(3,13)$. Research has confirmed that pyroptosis also significantly affects the progression of SLE, Faliti et al. found that restoring P2X7 activity in SLE patients could selectively limit the progressive amplification of pathogenic autoantibodies, which deteriorate patients' conditions $(14,15)$. NLR Family Pyrin Domain Containing 3 (NLRP3) inflammasome, an inflammasome sensor that mediates pyroptosis, was found to be hyperactivated in patients with SLE and LN (16). In the presence of antidouble-stranded DNA (dsDNA) antibodies, dsDNA can induce the activation of the NLRP3 inflammasome (17). Similarly, NLRP3 inflammasome activation can also be triggered by the interaction of U1-small nuclear ribonucleoprotein (U1-snRNP) and anti- U1-snRNP antibodies $(18,19)$. However, these studies only investigated the role of pyroptosis in SLE, and the process of pyroptosis is affected by many factors, such as pyroptosis-related genes. In this study, we examined pyroptosis-regulated genes to investigate the correlation between genes and SLE.

We present the following article in accordance with the STREGA reporting checklist (available at https://dx.doi. org/10.21037/atm-21-6193).

\section{Methods}

\section{Original data}

We downloaded three sets of gene microarrays (GSE11090, GSE20864, and GSE112087) from the Gene Expression Omnibus (GEO) database, including SLE and normal samples. After normalization and combination, a gene expression matrix of 122 normal samples and 239 SLE samples was obtained for further analysis. The study was conducted in accordance with the Declaration of Helsinki (as revised in 2013).

\section{The acquisition of DEGs}

An analysis of gene differences between the SLE and normal samples was performed using the LIMMA package in $\mathrm{R}$ software. $\mathrm{A} \log _{2}$ fold change $\left|\log _{\mathrm{FC}}\right|>0.5$ and a false discovery rate $(\mathrm{FDR})<0.05$ indicated that the genes were differentially expressed.

\section{Enrichment analysis of DEGs}

A gene set variation analysis (GSVA package) was conducted to analyse and identify significantly different Kyoto Encyclopedia of Genes and Genomes (KEGG) pathways that were activated or inhibited in the SLE sample. The gene oncology (GO) enrichment analysis of the DEGs was conducted using the ClusterProfiler package.

\section{Identifying the SLE-related pyroptosis gene}

Forty pyroptosis-related genes in the pyroptosis signaling pathway from the gene set enrichment analysis (GSEA, https://www.gsea-msigdb.org/) were extracted and intersected with the SLE DEGs. We also analyzed the 
expression of SLE-related pyroptosis genes in SLE and normal tissues, and their correlation with clinical traits.

\section{Immunological infiltration analysis}

We calculated the relative abundance of 22 immunocytes in the samples with the CIBERSORT algorithm, and analyzed the relationship between the immunocytes and SLE-related pyroptosis gene.

\section{Validation experiments}

Finally, we performed several validation experiments to confirm the finally selected gene expression levels between female and male SLE patients and normal patients.

\section{Statistical analysis}

All the statistical analyses were conducted using R software (version 4.0.3). The difference gene analysis used the LIMMA package. The following threshold was set: $\left|\log _{\mathrm{FC}}\right|$ $>0.5$ and FDR $<0.05$. The Wilcoxon non-parameter test was used to compare two groups. The Spearman method was used for the correlation analysis A P value $<0.05$ indicated a statistically significant difference.

\section{Results}

\section{DEGs and differential signaling pathways in SLE}

A total of 3,398 identical genes were obtained from the three data sets for the differential analysis. Figure $1 A$ shows a heatmap of the top 20 up/downregulated genes in SLE. Eighty-four upregulated genes and 52 downregulated genes were identified in SLE (see Figure 1B).

The enrichment analysis of the 136 DEGs revealed 10 significantly altered pathways, 6 of which were activated and 4 of which were inhibited in SLE (see Figure 1C).

\section{GO enrichment analysis of DEGs in SLE}

The biological process (BP) of the DEGs in SLE was mainly enriched in the defense response to the virus, the interferongamma-mediated signaling pathway, the type I interferon signaling pathway, the cellular response to type I interferon, and the response to type I interferon. The circle diagram shows the genes contained in the 5 BPs (see Figure $2 A$ ). The cell components were mainly enriched in the cell- substrate junction, focal adhesion, oxidoreductase complex, fibrillar center, and npBAF complex. The molecular function was mainly enriched in pattern recognition receptor activity, 4 iron, 4 sulfur cluster binding, NADH dehydrogenase activity, and phospholipid scramblase activity (see Figure 2B).

\section{Acquisition of the SLE-related pyroptosis gene}

We identified the SLE-related pyroptosis gene, ZBP1, by intersecting the DEGs of SLE with the 40 pyroptosis genes (see Figure $3 A$ ). The differential analysis indicated that ZBP1 was more highly expressed in SLE patients compared to normal samples $(\mathrm{P}<0.001$; see Figure $3 B)$. Additionally, the expression of $Z B P 1$ was higher in females than males $(\mathrm{P}=0.008$; see Figure 3C).

\section{Analysis of immune infiltration in SLE and normal samples}

The CIBERSORT algorithm was used to calculate 22 immunocells in 361 samples. Figure $4 A$ shows the immune cell abundance of all the samples. Figure $4 B$ shows the correlation between the 22 immunocell in the SLE patients, among them, resting dendritic cells and M2 macrophages had the highest negative correlations, and B memory cells and plasma were the most relevant (see Figure 4B). T cells CD8, monocytes, M1 macrophages, dendritic cells, eosinophils and neutrophils were more highly infiltrated in patients with SLE. While resting memory CD4 T cells, activated memory CD4 $\mathrm{T}$ cells, gamma delta $\mathrm{T}$ cells, and resting natural killer (NK) cells were lowly infiltrated in patients with SLE (see Figure 4C).

\section{The relationship between gender and immunocell abundance in SLE patients}

The immunocell abundance and gender difference analyses demonstrated that activated dendritic cells $(\mathrm{P}=0.039$, Figure $5 A), \mathrm{M} 1$ macrophages $(\mathrm{P}=0.019$, Figure $5 B)$, and neutrophils $(\mathrm{P}=0.0032$, Figure $5 C)$ were more highly infiltrated in female patients than male patients.

\section{The relationship between ZBP1 expression and immunocells}

The results indicated that activated immunocell dendritic cells and M2 macrophages were positively correlated 


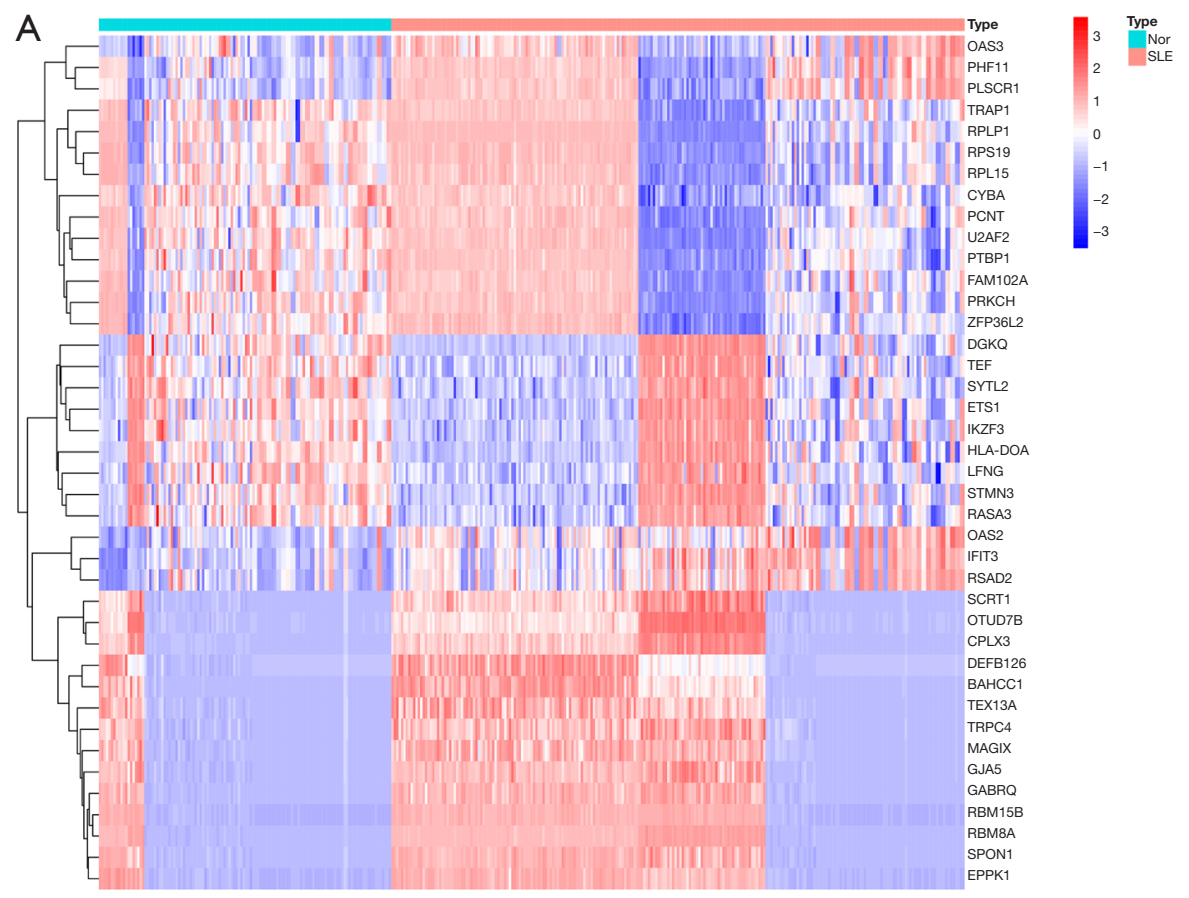

B

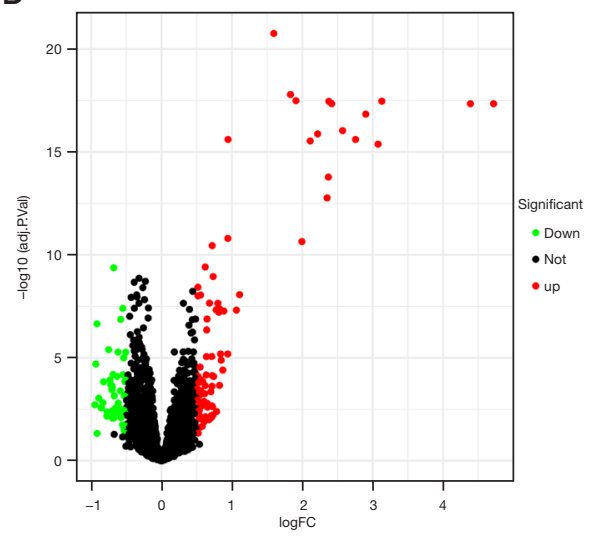

C

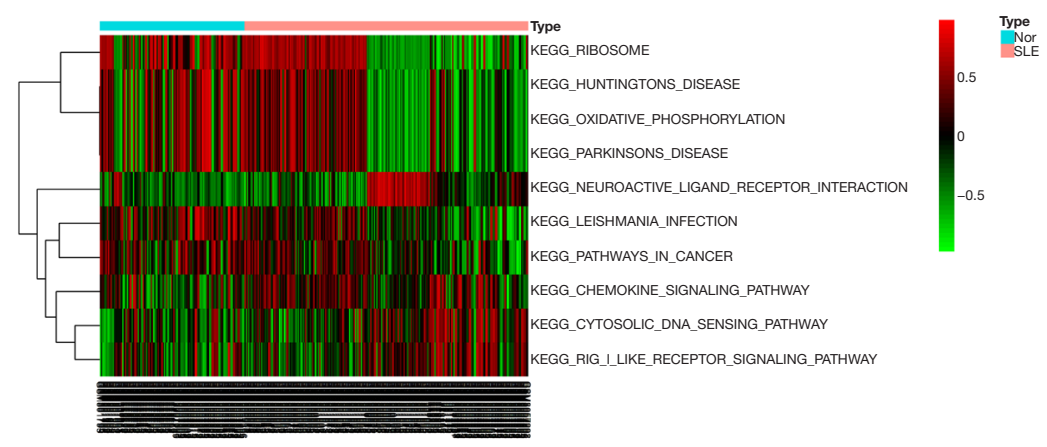

Figure 1 DEGs and differential signaling pathways in SLE. (A) Heatmap of the top 20 up/downregulated genes in SLE; (B) volcanic plot of DEGs in SLE; (C) heatmap of the dysregulated KEGG signaling pathways in SLE. DEGs, differentially expressed genes; KEGG, Kyoto encyclopedia of genes and genomes; SLE, systemic lupus erythematosus.

with the expression levels of $Z B P 1$, but resting dendritic cells and monocytes were negatively correlated with the expression levels of $Z B P 1$ in SLE patients (see Figure 6A-6D). Additionally, activated immunocell plasma cells, CD4 memory $\mathrm{T}$ cells, resting CD4 memory T cells, and naive CD4 T cells were positively correlated with $Z B P 1$ expression (see Figure 6E-6H); however, CD8 T cells, follicular helper T cells, gamma delta $\mathrm{T}$ cells, and regulatory $\mathrm{T}$ cells were positively correlated with ZBP1 expression (see Figure 6I-6L).

\section{Validation experiments}

To investigate the ZBP1 expression levels in SLE and normal samples, we performed western blotting and polymerase chain reaction analyses to validate the expression of $Z B P 1$, and the results showed that $Z B P 1$ was significantly more highly expressed in female and SLE patients than male and normal patients, respectively (see Figure $7 A-7 C)$. 
A

FAM111A

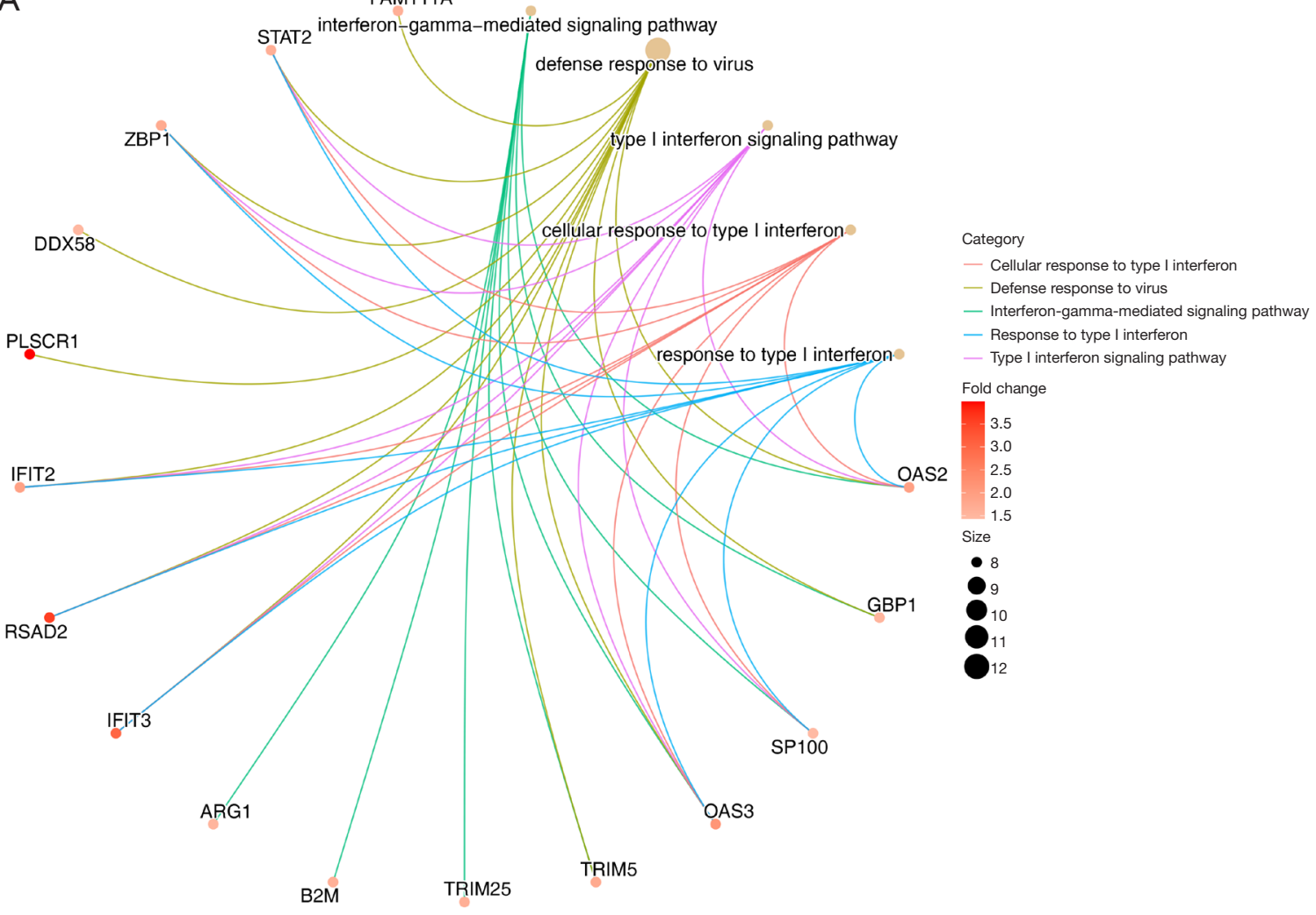

B

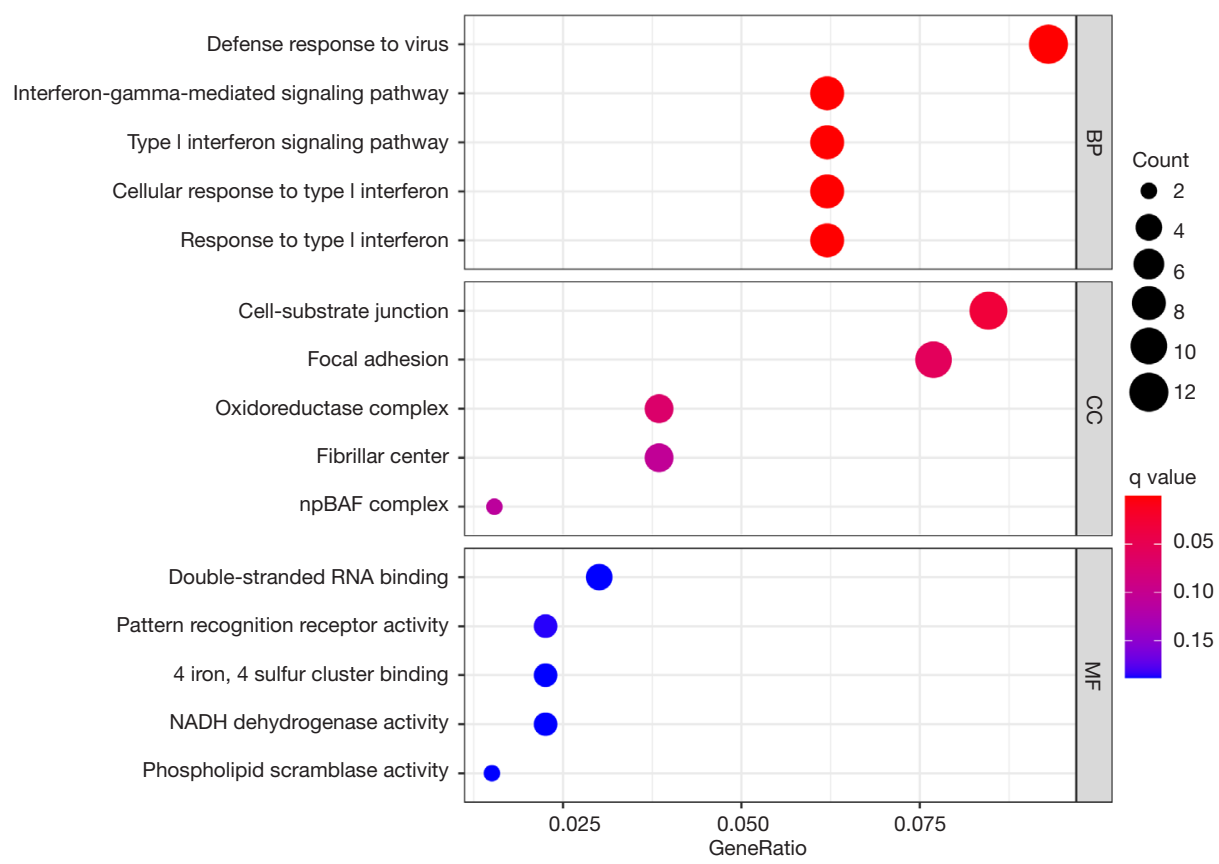

Figure 2 GO enrichment analysis of DEGs in SLE. (A) Circle diagram of the genes contained in the 5 BPs; (B) bubble diagram of the biological processes, cell components, and molecular functions of GO enrichment functions. BPs, biological processes, DEGs, differentially expressed genes; GO, gene oncology; SLE, systemic lupus erythematosus; BP, biological process; CC, cell components; MF, molecular function. 

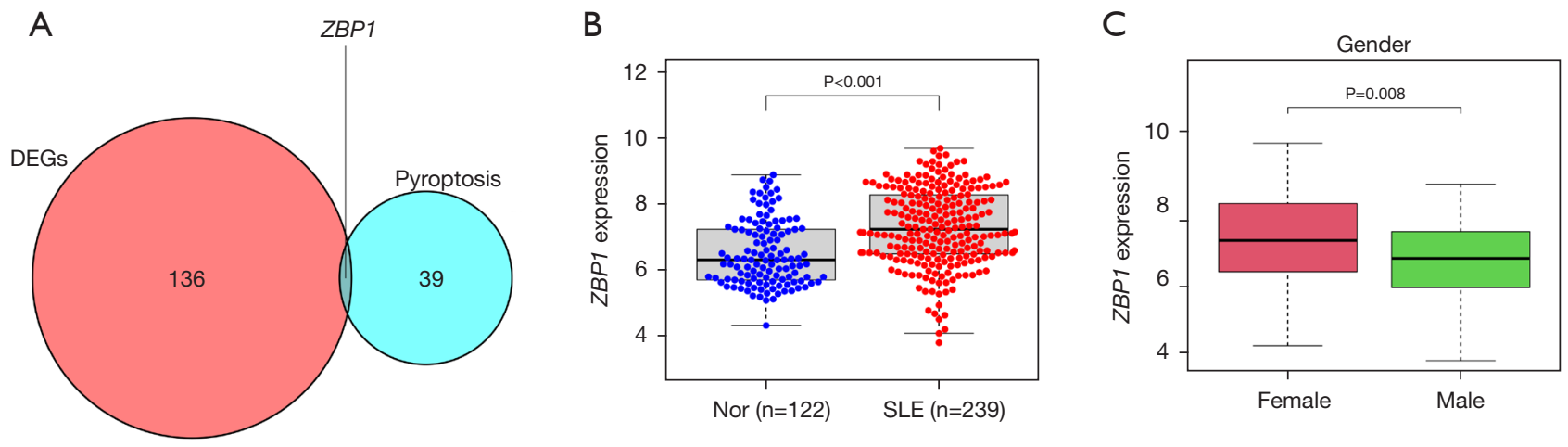

Figure 3 The expression and gender difference of the pyroptosis gene ZBP1 in SLE. (A) Venn diagram of DEGs of SLE and pyroptosis genes; (B) expression levels of ZBP1 in SLE blood samples and normal blood samples; (C) expression levels of ZBP1 in male and female SLE samples. DEGs, differential expressed genes; SLE, systemic lupus erythematosus; ZBP1, Z-DNA Binding Protein 1.

A

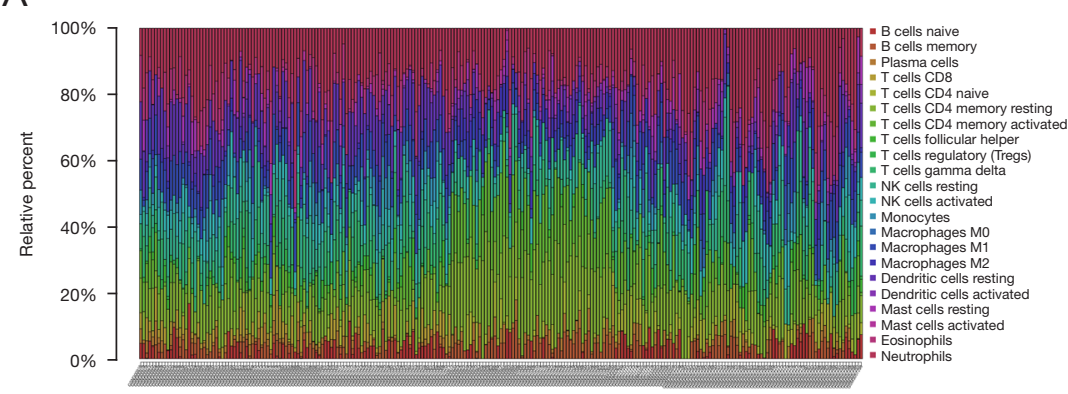

C

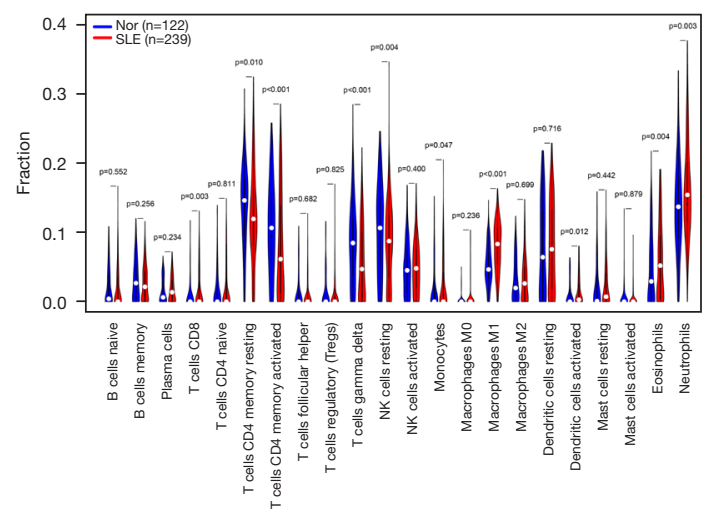

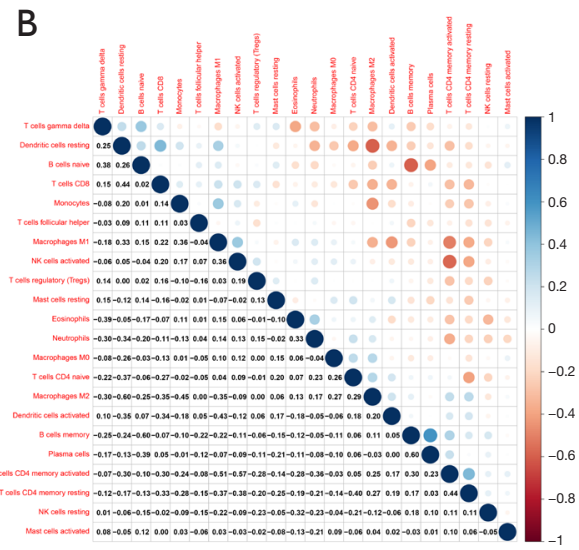

Figure 4 Analysis of immune infiltration in SLE and normal samples. (A) Histogram of the relative abundance of 22 immunocells in SLE and normal samples; (B) correlation heatmap between 22 immunocells; (C) Violin diagram of the differential expression of 22 immunocells between SLE and normal samples. SLE, systemic lupus erythematosus.

\section{Discussion}

SLE is an autoimmune disease, and it can damage several important organs, such as the brain, heart, kidneys, and lungs, and SLE patients suffer more during in these target organ complications. The epidemiology of SLE proves that it has a significant relationship with gender, and young women are at a higher risk than young men in terms of morbidity. Besides, the multiple underlying mechanisms of SLE have been classified. Among the various mechanisms, cell death plays a key role in SLE progression. The cell- 

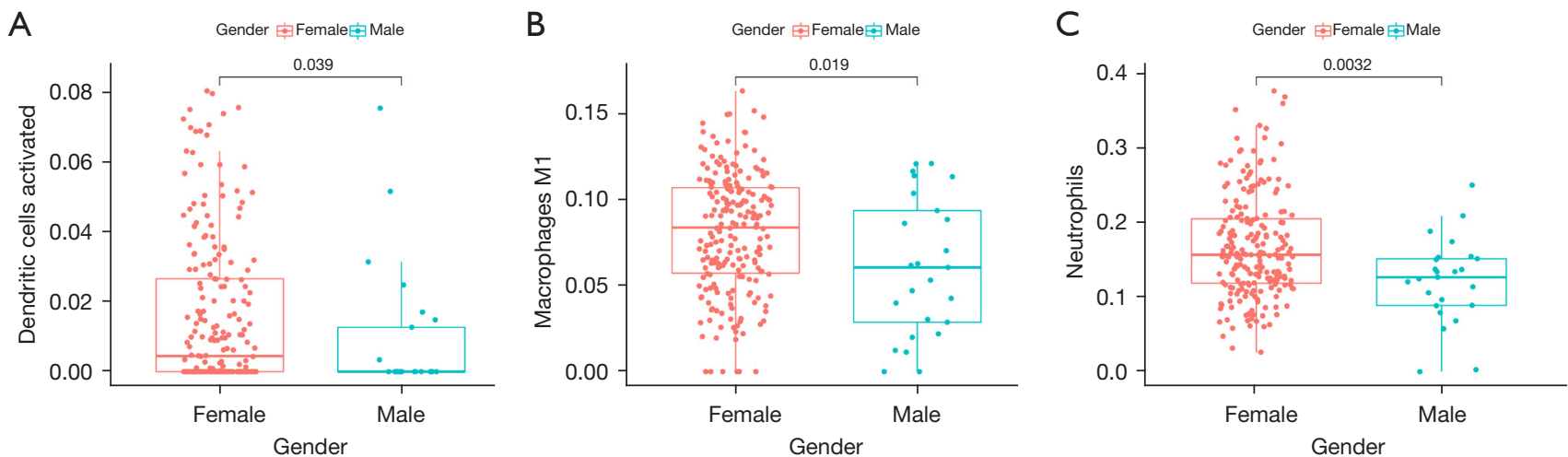

Figure 5 The relationship between gender and immunocell abundance in SLE patients. (A) Activated dendritic cells; (B) M1 macrophages; (C) neutrophils. SLE, systemic lupus erythematosus.
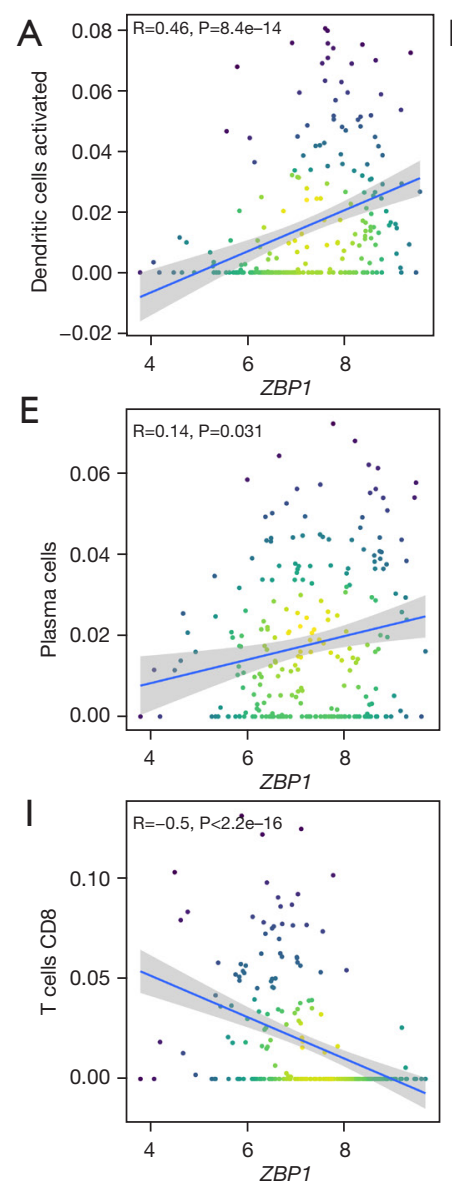
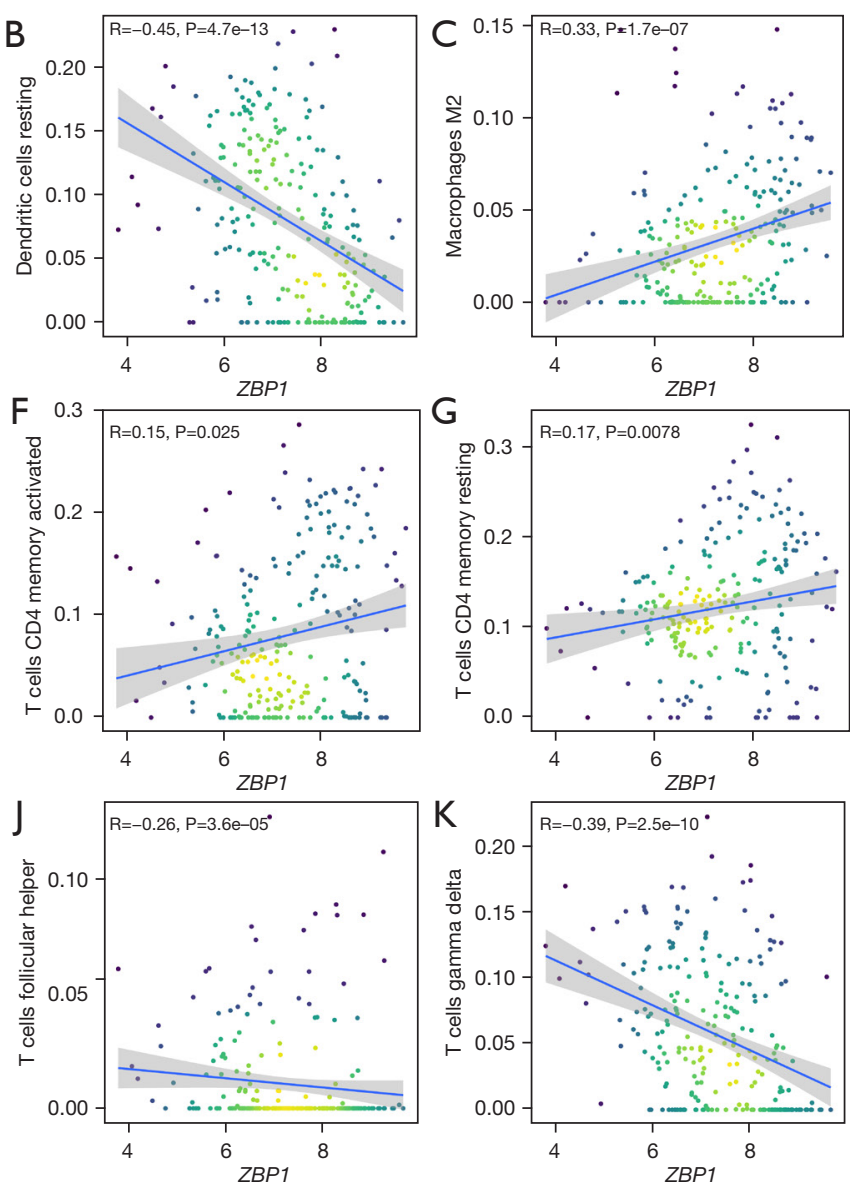
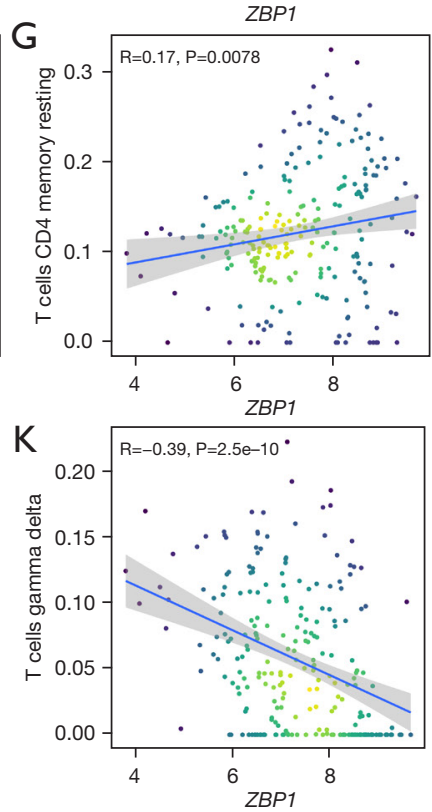
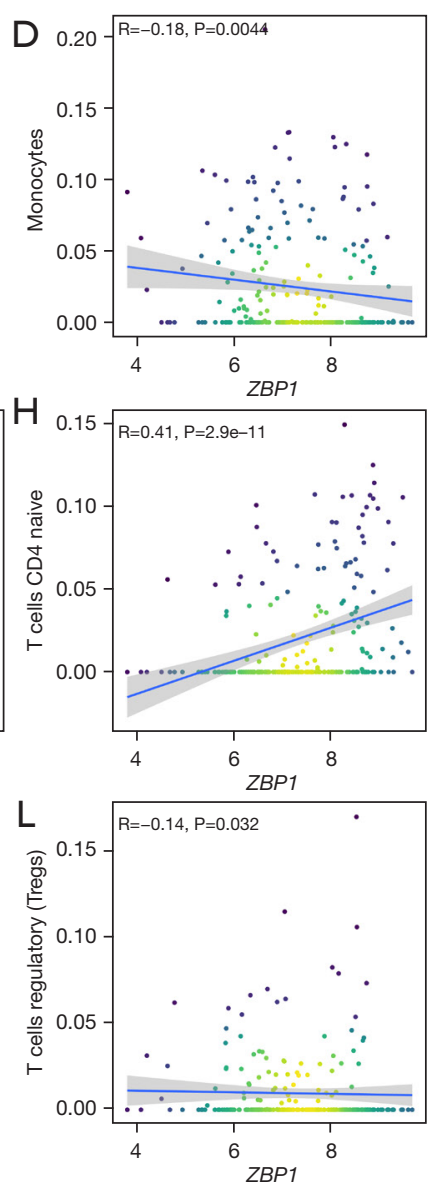

Figure 6 The relationship between ZBP1 expression and immunocells. (A) Activated dendritic cells; (B) resting dendritic cells; (C) M2 macrophages; (D) monocytes; (E) plasma cells; (F) activated CD4 memory T cells; (G) resting CD4 memory T cells; (H) CD4 naïve T cells; (I) CD8 T cells; (J) Follicular helper T cells; (K) Gamma delta T cells; (L) regulatory T cells. ZBP1, Z-DNA Binding Protein 1. 
A

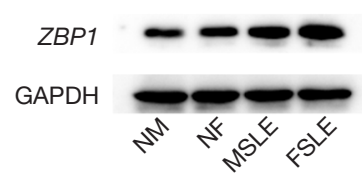

B

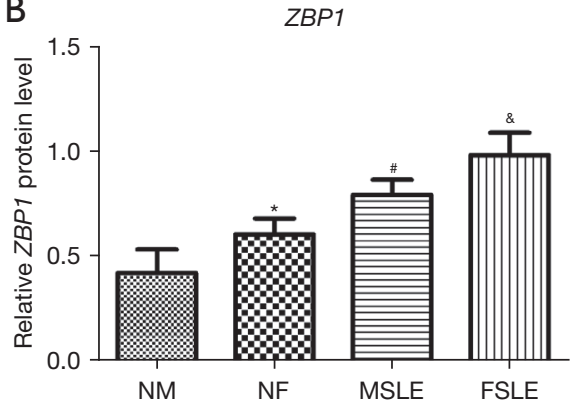

C

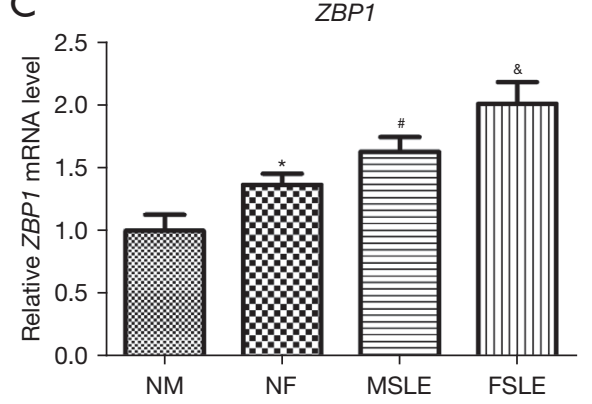

Figure 7 The validation experiments of $Z B P 1$. (A,B) The western blot of $Z B P 1$; (C) the quantitative real-time PCR of $Z B P 1$. * represent no-SLE female samples; ${ }^{*}$ represent male SLE patients samples, and ${ }^{\&}$ represent female SLE samples, respectively. ZBP1, Z-DNA Binding Protein 1; PCR, polymerase chain reaction.

type process induces inflammation, releases auto antigens, and leads to some diseases. Research of the pyroptosis process revealed that it is involved in multiple diseases, including SLE (20-22). This study explored pyroptosis gene expression levels in different samples, the fundamental biological functions of pyroptosis' DEGs, and pyroptosis gene' role in SLE.

In present study, the results showed that 136 DEGs were differentially expressed between the SLE and normal samples. The top DEGs included OAS3, PHF11, PLSCR1, TRAP1, RPLP1, RPS19, RPL15, CYBA, PCNT, U2AF2, $P T B P 1$. Gao et al. revealed MALAT1 affects SLE by regulating the expression of OAS3 (23). Yamada et al. found that PHF11 is a regulator of inflammation (24). PPLSCR1 was observed to be overexpressed in monocytes in SLE samples (25). TRAP1 and RPLP1 play key roles in SLE progression $(26,27)$. Regrettably, several genes have not been found to have an association with SLE, including OAS3, PHF11, PPLSCR1, TRAP1, and RPLP1. In the present study, we examined the enrichment functions of these DEGs, and found that NADH dehydrogenase activity, phospholipid scramblase activity, double-stranded RNA binding, and the interferon signaling pathway may be the most important biological functions. Suzuki et al. found that phospholipid scramblase (phospholipid scramblase 1) induces fibrin turnover and increases cell-surface phosphatidylserine exposure, promoting the risk of thrombus (25). Doublestranded RNA antibodies have also been investigated in SLE, and RNA antibody levels have been found to be associated with disease activity (28). Interferon has been shown to act as an important regulator of SLE, and interferon lambda affects the immune system and inflammation dysregulation (29).

Thus, DEGs play a key role in SLE. We selected pyroptosis genes for further analysis and examined the intersection between the pyroptosis genes and DEGs in the SLE samples. ZBP1 was selected and we investigated its role in SLE. The results showed that ZBP1 was significantly more highly expressed in SLE patients than normal patients, and the subgroup analysis revealed that females have higher $Z B P 1$ expression levels than males. $Z B P 1$ is a $Z$-DNA-binding protein type that can be induced via interferons (30). ZBP1 can act as a regulator of interferoninduced necroptosis (31). Further, $Z B P 1$ is also an innate sensor for inflammation, and is a significant regulator of multiple disease processes, such as influenza virus infection, NLRP3 inflammasome, and proinflammatory responses $(32,33)$. Takaoka et al. revealed that $Z B P 1$ is the ligand of double-strain DNA, and may have an association with autoimmune disease (34). However, the role of $Z B P 1$ in SLE is still unclear.

As autoimmune disease and $Z B P 1$ have a significant relationship with inflammation and necroptosis, and immune cells act as important factors in these processes, we investigated the relationship between $Z B P 1$ and immune cell infiltration. The analysis results showed that $Z B P 1$ is significantly associated with several types of immune cell infiltration, including dendritic cell, monocyte, macrophage, CD4 T cell, and CD8 T cell infiltration. $Z B P 1$ is negatively correlated with regulatory $\mathrm{T}$ cells, and many studies have shown regulatory $\mathrm{T}$ cells play a key role in SLE progression (35-37). Several studies have revealed that SLE patients have increased CD8 T cell levels, which induce autoantibody production, resulting in organ damage $(38,39)$. However, in the present study, ZBP1 was found to be negatively correlated with CD8 T cell infiltration.

Macrophage cells are critical cells in the inflammation 
process and autoimmune disease. Macrophages are tissue resident immune cells that can as immune respond cell. Macrophages canactivated via IgG IC, induce the production of cytokines, such as IL- $6, \mathrm{TNF} \alpha$, IL-1 $\beta$, and inflammatory mediators (40-42). Jing et al. revealed that macrophages may be reprogrammed as therapy targets for LN (43). The biological function of monocytes in SLE has also been explored. Monocyte cells can induce change via cytokines and have a significant effect on SLE (44). Additionally, $\mathrm{CD}^{+}{ }^{+} \mathrm{T}$ cells, B cells, and plasma cells also have a strong effect on the progression of SLE (45-47). These results showed that $Z B P 1$ can play a significant role by regulating various immune cell infiltration in SLE patients.

\section{Conclusions}

In the present study, we found that the pyroptosis gene, $Z B P 1$, may act as a biomarker for diagnosing and assessing the activity of SLE. Our findings may lead to the development of complementary therapy methods for SLE via the regulation of pyroptosis genes, such as $Z B P 1$.

\section{Acknowledgments}

Funding: None.

\section{Footnote}

Reporting Checklist: The authors have completed the STREGA reporting checklist. Available at https://dx.doi. org/10.21037/atm-21-6193

Conflicts of Interest: All authors have completed the ICMJE uniform disclosure form (available at https://dx.doi. org/10.21037/atm-21-6193). The authors have no conflicts of interest to declare.

Ethical Statement: The authors are accountable for all aspects of the work in ensuring that questions related to the accuracy or integrity of any part of the work are appropriately investigated and resolved. The study was conducted in accordance with the Declaration of Helsinki (as revised in 2013).

Open Access Statement: This is an Open Access article distributed in accordance with the Creative Commons Attribution-NonCommercial-NoDerivs 4.0 International
License (CC BY-NC-ND 4.0), which permits the noncommercial replication and distribution of the article with the strict proviso that no changes or edits are made and the original work is properly cited (including links to both the formal publication through the relevant DOI and the license). See: https://creativecommons.org/licenses/by-nc-nd/4.0/.

\section{References}

1. Tsokos GC. Systemic lupus erythematosus. N Engl J Med 2011;365:2110-21.

2. Pisetsky DS. Anti-DNA antibodies--quintessential biomarkers of SLE. Nat Rev Rheumatol 2016;12:102-10.

3. Magna M, Pisetsky DS. The Role of Cell Death in the Pathogenesis of SLE: Is Pyroptosis the Missing Link? Scand J Immunol 2015;82:218-24.

4. Kerr JF, Wyllie AH, Currie AR. Apoptosis: a basic biological phenomenon with wide-ranging implications in tissue kinetics. Br J Cancer 1972;26:239-57.

5. Álvarez K, Vasquez G. Damage-associated molecular patterns and their role as initiators of inflammatory and auto-immune signals in systemic lupus erythematosus. Int Rev Immunol 2017;36:259-70.

6. Kovacs SB, Miao EA. Gasdermins: Effectors of Pyroptosis. Trends Cell Biol 2017;27:673-84.

7. Zychlinsky A, Prevost MC, Sansonetti PJ. Shigella flexneri induces apoptosis in infected macrophages. Nature 1992;358:167-9.

8. Próchnicki T, Mangan MS, Latz E. Recent insights into the molecular mechanisms of the NLRP3 inflammasome activation. F1000Res 2016;5:eF1000 Faculty Rev-1469.

9. Fang $\mathrm{Y}$, Tian S, Pan Y, et al. Pyroptosis: A new frontier in cancer. Biomed Pharmacother 2020;121:109595.

10. Guo Q, Wu Y, Hou Y, et al. Cytokine Secretion and Pyroptosis of Thyroid Follicular Cells Mediated by Enhanced NLRP3, NLRP1, NLRC4, and AIM2 Inflammasomes Are Associated With Autoimmune Thyroiditis. Front Immunol 2018;9:1197.

11. Jia C, Chen H, Zhang J, et al. Role of pyroptosis in cardiovascular diseases. Int Immunopharmacol 2019;67:311-8.

12. Xu YJ, Zheng L, Hu YW, et al. Pyroptosis and its relationship to atherosclerosis. Clin Chim Acta 2018;476:28-37.

13. Pieterse E, van der Vlag J. Breaking immunological tolerance in systemic lupus erythematosus. Front Immunol 2014;5:164.

14. Wu D, Ai L, Sun Y, et al. Role of NLRP3 Inflammasome 
in Lupus Nephritis and Therapeutic Targeting by Phytochemicals. Front Pharmacol 2021;12:621300.

15. Faliti CE, Gualtierotti R, Rottoli E, et al. P2X7 receptor restrains pathogenic $\mathrm{Tfh}$ cell generation in systemic lupus erythematosus. J Exp Med 2019;216:317-36.

16. Mistry P, Kaplan MJ. Cell death in the pathogenesis of systemic lupus erythematosus and lupus nephritis. Clin Immunol 2017;185:59-73.

17. Sharma M, de Alba E. Structure, Activation and Regulation of NLRP3 and AIM2 Inflammasomes. Int J Mol Sci 2021;22:872.

18. Shin MS, Kang Y, Lee N, et al. U1-small nuclear ribonucleoprotein activates the NLRP3 inflammasome in human monocytes. J Immunol 2012;188:4769-75.

19. Shin MS, Kang Y, Wahl ER, et al. Macrophage Migration Inhibitory Factor Regulates U1 Small Nuclear RNP Immune Complex-Mediated Activation of the NLRP3 Inflammasome. Arthritis Rheumatol 2019;71:109-20.

20. Vandanmagsar B, Youm YH, Ravussin A, et al. The NLRP3 inflammasome instigates obesity-induced inflammation and insulin resistance. Nat Med 2011;17:179-88.

21. Yuan Y, Liu Z. Isoflurane attenuates murine lupus nephritis by inhibiting NLRP3 inflammasome activation. Int J Clin Exp Med 2015;8:17730-8.

22. Zhao J, Wang H, Dai C, et al. P2X7 blockade attenuates murine lupus nephritis by inhibiting activation of the NLRP3/ASC/caspase 1 pathway. Arthritis Rheum 2013;65:3176-85.

23. Gao F, Tan Y, Luo H. MALAT1 is involved in type I IFNs-mediated systemic lupus erythematosus by upregulating OAS2, OAS3, and OASL. Braz J Med Biol Res 2020;53:e9292.

24. Yamada R, Ymamoto K. Recent findings on genes associated with inflammatory disease. Mutat Res 2005;573:136-51.

25. Suzuki E, Amengual O, Atsumi T, et al. Increased expression of phospholipid scramblase 1 in monocytes from patients with systemic lupus erythematosus. J Rheumatol 2010;37:1639-45.

26. Hu C, Huang W, Chen $\mathrm{H}$, et al. Autoantibody profiling on human proteome microarray for biomarker discovery in cerebrospinal fluid and sera of neuropsychiatric lupus. PLoS One 2015;10:e0126643.

27. Li S, Sun X, Xu J, et al. Association study of TRAP1 gene polymorphisms with susceptibility and glucocorticoids efficacy of systemic lupus erythematosus. Gene 2018;671:117-26.
28. Davis P, Cunnington P, Hughes GR. Double-stranded RNA antibodies in systemic lupus erythematosus. Ann Rheum Dis 1975;34:239-43.

29. Goel RR, Wang X, O'Neil LJ, et al. Interferon lambda promotes immune dysregulation and tissue inflammation in TLR7-induced lupus. Proc Natl Acad Sci U S A 2020;117:5409-19.

30. Fu Y, Comella N, Tognazzi K, et al. Cloning of DLM-1, a novel gene that is up-regulated in activated macrophages, using RNA differential display. Gene 1999;240:157-63.

31. Yang D, Liang Y, Zhao S, et al. ZBP1 mediates interferoninduced necroptosis. Cell Mol Immunol 2020;17:356-68.

32. Kuriakose T, Man SM, Malireddi RK, et al. ZBP1/DAI is an innate sensor of influenza virus triggering the NLRP3 inflammasome and programmed cell death pathways. Sci Immunol 2016;1:aag2045.

33. Thomas PG, Dash P, Aldridge JR Jr, et al. The intracellular sensor NLRP3 mediates key innate and healing responses to influenza A virus via the regulation of caspase-1. Immunity 2009;30:566-75.

34. Takaoka A, Wang Z, Choi MK, et al. DAI (DLM-1/ ZBP1) is a cytosolic DNA sensor and an activator of innate immune response. Nature 2007;448:501-5.

35. Geginat J, Vasco M, Gerosa M, et al. IL-10 producing regulatory and helper T-cells in systemic lupus erythematosus. Semin Immunol 2019;44:101330.

36. Giang S, La Cava A. Regulatory T Cells in SLE: Biology and Use in Treatment. Curr Rheumatol Rep 2016;18:67.

37. Tower C, Crocker I, Chirico D, et al. SLE and pregnancy: the potential role for regulatory $T$ cells. Nat Rev Rheumatol 2011;7:124-8.

38. Denny MF, Chandaroy P, Killen PD, et al. Accelerated macrophage apoptosis induces autoantibody formation and organ damage in systemic lupus erythematosus. J Immunol 2006;176:2095-104.

39. Kaplan MJ, Lu Q, Wu A, et al. Demethylation of promoter regulatory elements contributes to perforin overexpression in CD4+ lupus T cells. J Immunol 2004;172:3652-61.

40. Clatworthy MR, Smith KG. FcgammaRIIb balances efficient pathogen clearance and the cytokine-mediated consequences of sepsis. J Exp Med 2004;199:717-23.

41. Floto RA, Clatworthy MR, Heilbronn KR, et al. Loss of function of a lupus-associated FcgammaRIIb polymorphism through exclusion from lipid rafts. Nat Med 2005;11:1056-8.

42. Guilliams M, Bruhns P, Saeys Y, et al. The function of Fcgamma receptors in dendritic cells and macrophages. 
Nat Rev Immunol 2014;14:94-108.

43. Jing C, Castro-Dopico T, Richoz N, et al. Macrophage metabolic reprogramming presents a therapeutic target in lupus nephritis. Proc Natl Acad Sci U S A 2020;117:15160-71.

44. Zhang Z, Maurer K, Perin JC, et al. Cytokine-induced monocyte characteristics in SLE. J Biomed Biotechnol 2010;2010:507475.

Cite this article as: Huang Y, Yang DD, Li XY, Fang DL, Zhou WJ. ZBP1 is a significant pyroptosis regulator for systemic lupus erythematosus. Ann Transl Med 2021;9(24):1773. doi: 10.21037/atm-21-6193
45. Clarke J. IL-17 sustains plasma cells in SLE. Nat Rev Rheumatol 2020;16:666.

46. Tsubata T. Inhibitory B cell co-receptors and autoimmune diseases. Immunol Med 2019;42:108-16.

47. Yin Y, Choi SC, Xu Z, et al. Normalization of CD4+ T cell metabolism reverses lupus. Sci Transl Med 2015;7:274ra18.

(English Language Editor: L. Huleatt) 